Pacific Journal of Mathematics

REPRESENTATIONS OF DIVISION ALGEBRAS OVER LOCAL 


\section{REPRESENTATIONS OF DIVISION ALGEBRAS OVER LOCAL FIELDS: II}

\section{LAWRENCE CORWIN}

Let $D$ be a division algebra of degree $p^{4}$ over a nonArchimedean locally compact field $K$ (i.e., index $(D / K)=p^{2}$ ). We show how to construct the irreducible unitary representations of the multiplicative group of $D$.

1. Let $K$ be a non-Archimedean locally compact field, with residue field $k$ having $q=p^{r}$ elements ( $p$ is a prime), and let $D$ be a locally compact division algebra with center $K$. Then $[D: K]$ is a perfect square $-m^{2}$, say; $m$ is called the index of $D$. It is a question of some interest to determine the irreducible unitary representations of $D^{x}$. In [1], [2], and [3], the representations were determined and their characters computed for the case where $m$ is prime to $p$ (the "tamely ramified" case). As was pointed out in [4], the same procedure serves to compute the representations (though not the characters) in the case $m=p$.

In this paper we compute the irreducible representations of $D^{x}$ in the case where $m=p^{2}$. The procedure is like that of the previous cases, but there are added technical difficulties. The method as given here does work in somewhat greater generality; see $\S 5$ for a brief discussion.

Before describing the method, we need some notation. Let $K_{1}$ be a maximal unramified extension of $K$ contained in $D$, so that $\left[K_{1}: K\right]=m$; let $d$ be the residue class field of $K_{1}$ (and of $D$ ). It is possible to pick a prime element $\pi$ of $D$ such that conjugation by $\pi$ generates $\mathrm{Gal}\left(K_{1} / K\right)$. (For these and other facts used here, see, e.g., Chapter 1 of [8].) Then $\pi^{m}$ is a prime element of $K$. We may also choose a set $k_{1} \subset K_{1}$ of coset representatives of $d$ such that $0 \in k_{1}$ and the other elements of $k_{1}$ are the $\left(q^{m}-1\right)$ th roots of unity in $K_{1}$. Then every element of $D$ has a unique expression of the form

$$
y=\sum_{j=j_{0}}^{\infty} \alpha_{j} \pi^{j}, \quad j_{0} \in Z,
$$

where each $\alpha_{j} \in k_{1}$. Set

$$
D_{1}=\left\{y=\sum_{j=0}^{\infty} \alpha_{j} \pi^{j}: \alpha_{0} \neq 0\right\}
$$

and

$$
G=\left\{y \in D_{1}: \alpha_{0}=1\right\} \text {. }
$$


Then

$$
D^{\mathbf{x}}=D_{1} \ltimes\left\{\pi^{j}: j \in Z\right\}
$$

and

$$
D_{1}=G \ltimes k_{1}^{\prime}, \quad k_{1}^{\prime}=k_{1} \backslash\{0\} .
$$

As noted in [1], the major problem in finding irreducible representations of $D^{x}$ lies in finding those of $G$.

Let $\mathfrak{O}=\mathfrak{D}_{D}=\left\{y=\sum_{j=0}^{\infty} \alpha_{j} \pi^{j}\right\}$ be the ring of integers of $D$, and

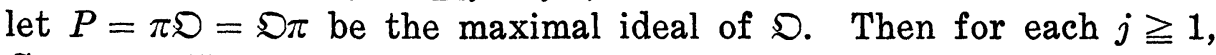
$G_{j}=1+P^{j}$ is a subgroup of $G$ (and $G=G_{1}$ ). Any irreducible representation $\rho$ of the compact group $G$ must be trivial on some $G_{j}$; let $n$ be the smallest integer ( $\geqq 1)$ such that $\rho \mid G_{n+1}$ is trivial. (In the terminology of [3], $n+1$ is the conductor of $\rho$.) Let $n_{0}=[(n+1) / 2]$, where [ ] denotes the greatest integer function. Then $G_{n_{0}} / G_{n} \cong$ $P^{n_{0}} / P^{n+1}$ is Abelian, and therefore $\rho \mid G_{n_{0}}$ is a direct sum of 1 -dimensional representations. Let $\chi$ be one of these, and regard $\chi$ as a representation of $P^{n_{0}}$. We may describe $\chi$ as follows: let $\psi$ be a character of $K^{+}$which is trivial on $\mathfrak{D}_{K}=\mathfrak{D}_{D} \cap K$, but not on $\pi^{-m} \mathfrak{D}_{K}$. Then any character of $D^{+}$is of the form

$$
\chi_{x}(y)=\psi \circ \tau(x y),
$$

where $x \in D$ and $\tau: D \rightarrow K$ is the reduced trace. If we identify $D$ with $D^{\wedge}$ via $x \leftrightarrow \chi_{x}$, then $\mathfrak{D}_{\bar{D}}^{\perp}=P^{-m+1}$ and $\left(P^{n+1}\right)^{\perp}=P^{-m-n},\left(P^{n_{0}}\right)^{\wedge}=D / P^{-m-n_{0}+1}$. The character $\chi$ is an element of $\left(P^{n_{0}}\right)^{\wedge}$ that annihilates $P^{n+1}$, and is thus given by an element of $P^{-m-n} / P^{-m-n_{0}+1}$. That is, $\chi=\chi_{x}$, where $x \in P^{-m-n}$ (and $x$ is determined $\bmod P^{-m-n_{0}+1}$ ). Conversely, $\rho$ appears in $\operatorname{Ind}_{G_{n_{0} \rightarrow G}}\left(\chi_{x}\right)$, and we therefore determine all irreducibles $\rho$ containing $\chi$ by finding all irreducible components of $\operatorname{Ind}_{\theta_{n_{0}} \rightarrow G} \chi_{x}$. Replacing $\chi_{x}$ by $\chi_{x^{\prime}}$, where $x$ and $x^{\prime}$ are conjugate in $G$, does not change the induced representation; if $x$ and $x^{\prime}$ are conjugate in $D$, the induced representation may be changed, but in an unimportant way so far as representations of $D$ are concerned.

The construction of $\rho$ in the tamely ramified case proceeds as follows: one first chooses $x$ to generate a field of degree as small as possible. Then one constructs a subgroup $H=H_{x}$ of $G$ which contains $G_{n_{0}}$ (roughly speaking, $H$ consists of elements of $G$ commuting with $x \bmod$ an appropriate power of $P$ ), extends $\chi_{x}$ to an irreducible representation $\chi_{x, a}$ of $H_{x}$, and induces up to $G$; the induced representation is irreducible, and one obtains all the irreducibles in $\operatorname{Ind}_{G_{n_{0} \rightarrow G}} \chi_{x}$ by varying the $\chi_{x ; a}$. The procedure in our case is similar, but in some cases the group $H_{x}$ depends on the extended representation $\chi_{x ; a}$.

The wildly ramified case seems to be harder than the tamely 
ramified one for two related reasons. Write $x=\sum_{j=-m-n}^{\infty} \alpha_{j} \pi^{j}, x_{1}=$ $\alpha_{-m-n} \pi^{-m-n}$. In the tamely ramified case, we may assume (by taking a conjugate of $x$ ) that the elements $\alpha_{j} \pi^{j}$ all commute. Futhermore, an important part in the analysis is played by elements $x_{i}$ which are conjugate to $x \bmod P^{-m-n+i}$ and which generate fields of degrees as small as possible. In the tamely ramified case, these elements can be assumed to lie in the division algebra $D_{x_{1}}$ of elements commuting with $x_{1}$, and therefore many proofs can proceed inductively. In the wildly ramified case, it is quite possible that $1<\left[K\left(x_{1}\right): K\right]<$ $[K(x): K]$ although $K(x)$ contains no proper extension of $K$. As a result, we need some technical lemmas to show that $D_{x_{1}}$ has elements "close" (in $D$ ) to those of $K(x)$. When these technical difficulties do not arise, the proofs are easier; we discuss these cases in $\S 2$.

For the rest of the paper, we set $m=p^{2}$ (though for typographical convenience we continue to use $m$ ). Let $K_{0} \subseteq K_{1}$ be an unramified extension of $K$ of order $p$.

In what follows, $\sigma$ denotes the element of $\mathrm{Gal}\left(K_{1} / K\right)$ generated by conjugation by $\pi ; \sigma$ is generally written exponentially. We also use $\sigma$ to denote the corresponding element of $\mathrm{Gal}(d / k)$. We shall often denote an element of $k_{1}$ and the corresponding element of $d$ by the same letter; for example, we shall write $\operatorname{Tr}_{d / k} \alpha$ for $\alpha \in k_{1}$. This convention should cause no confusion; in case Char $K=p$, it is justified by the fact that $d \cong k_{1}$. Similarly, we write $k, k_{0}$ for $K \cap k_{1}$, $K_{0} \cap k_{1}$, and regard elements of, e.g., $k$ as either in $K$ or in the residue class field. For $0,1 \neq x \in D, D_{x}$ is the division algebra of elements commuting with $x$; however, $D_{0}$ will be the division algebra of elements commuting with $K_{0}$. Note that if $[K(x): K]=m_{0}$, then the index of $D_{x}$ over $K(x)$ is $\left(m / m_{0}\right)^{2}$. Then reduced trace (norm) is denoted by $\tau(\nu)$. Finally, we shall write " $\rho$ contains $\chi$ " for "the restriction to $\left(1+P^{n_{0}}\right)$ of the irreducible representation $\rho$ of $G$ contains $\chi . "$

2. The results of this section are, generally speaking, either specifically contained in those of [1], [2], or [3], or easily deduced from them.

We continue with the notation of the first section. Let $\chi=\chi_{x}$, $x=\sum_{j=-n-m}^{\infty} \alpha_{j} \pi^{j}$, and set $x_{1}=\alpha_{-m-n} \pi^{-m-n}$. We assume throughout this section and the next two that $n$ is odd.

LEMMA 1. If $y=\sum_{j=-m-n}^{\infty} \beta_{j} \pi^{j}$, with $\beta_{-m-n}=\alpha_{-m-n}$, then $e(K(y)) \geqq$ $e\left(K\left(x_{1}\right)\right)$ and $f(K(y)) \geqq f\left(K\left(x_{1}\right)\right)$, where $e=$ ramification index and $f=$ residue class degree.

Proof. As the extra effort involved is small, we give a proof 
valid for all $m$. Write $\alpha$ for $\alpha_{-m-n}$. Since $\pi^{-m} \in K, K\left(x_{1}\right)=K\left(\alpha \pi^{-n}\right)=$ $K\left(x_{0}\right)$, say. Let $m=s t$, where $s$ is the greatest common divisor of $m$ and $n ; x_{0}^{t} \in K_{1}$. Let $K^{\prime}=K\left(x_{0}^{t}\right)$. Then $f\left(K\left(x_{1}\right)\right)=\left[K^{\prime}: K\right]$ and $e\left(K\left(x_{1}\right)\right)=t$.

Suppose that $a m-b n=s$. Then $\pi^{m(a+b)} y^{b} \in P^{s}$, so that $K(y)$ certainly has an element $y_{0}$ such that $\pi^{-m} y_{0}^{t}$ is a unit, and hence $e(K(y))$ is a multiple of $t$. Furthermore, $y^{t} \equiv x_{1}^{t} \bmod P^{-t(m+n)+1}$; now Hensel's lemma shows that $K(y)$ contains a field isomorphic to $K^{\prime}$. Thus $f(K(y)) \geqq\left[K^{\prime}: K\right]$, as claimed.

We now proceed to look at the representations of $G$. The analysis splits into four basic cases, depending on the nature of $x_{1}$ :

(1) $x_{1} \in K$

(2) $\left[K\left(x_{1}\right): K\right]=m$;

(3) $\left[K\left(x_{1}\right): K\right]=p$ and $K\left(x_{1}\right)$ is unramified over $K$;

(4) $\left[K\left(x_{1}\right): K\right]=p$ and $K\left(x_{1}\right)$ is totally ramified over $K$.

We deal with the first three cases in this section. Case (4), which splits into three subcases, is the subject of the next two sections.

Case (1) is handled by reducing it to the other cases; see [1], [3], or [4].

THEOREM 1. If $K\left(x_{1}\right)=K$, then every irreducible representation $\rho$ containing $\chi_{x}$ is of the form $\rho=\rho_{1} \otimes\left(\chi_{0} \circ \nu\right)$, where $\nu$ is the reduced norm map (from $D$ to $K$ ), $\chi_{0}$ is a 1-dimensional representation of $K^{x}$, and $\rho_{1}$ is a representation of $G$ with conductor $<n$.

Proof. If $x_{1} \in K$ and $1+y \in G_{n}$, then

$$
\chi_{x}(1+y)=\psi \circ \tau_{D / K}(x y)=\psi\left(x \tau_{D / K} y\right) .
$$

But $\nu(1+y) \equiv 1+\tau_{D / K}(y) \bmod G_{n+1}$, so that $\chi_{x}(1+y)$ depends only on $\nu(y)$. Hence there is a character $\chi_{0}$ of $K G$ such that $\chi=\chi_{0} \circ \nu$ on $G_{n}$. A simple counting argument (given in [1]) shows that $\operatorname{Ind}_{G_{n-1} \rightarrow G}\left(\left.\chi\right|_{G_{n-1}}\right)$ consists of multiples of representations $\rho_{1} \otimes\left(\chi_{0} \circ \nu\right)$, where $\rho_{1}$ has conductor $<n$. This proves the theorem.

Case (2) is also easy to deal with; the analysis follows that in $\S 4$ of [4] (or that of [1], Theorem 2.3).

LEMmA 2. If $\left[K\left(x_{1}\right): K\right]=m$ and $y \in K(x), y=\sum_{j=r}^{\infty} \beta_{j} \pi^{j}$, then $\beta_{r} \pi^{r} \in K\left(x_{1}\right)$; conversely, if $\beta_{r} \pi^{r} \in K\left(x_{1}\right)$, then $\exists y \in K(x)$ with $y=$ $\sum_{j=r}^{\infty} \beta_{r} \pi^{r}$.

Proof. Since $x y=y x$, the lowest order terms of $x$ and $y$ must commute-that is, if $y_{1}=\beta_{r} \pi^{r}$, then $y_{1} x_{1}=x_{1} y_{1}$. Thus $y_{1} \in D_{x_{1}}$. Since $K\left(x_{1}\right)$ is its own commutator in $D, y \in K\left(x_{1}\right)$. For the converse, fix $r$ 
and let $h^{\prime}=\left\{\beta \in k_{1}: \exists y \in K(x)\right.$ with $y=\sum_{j=r}^{\infty} \beta_{j} \pi^{j}$ and $\left.\beta_{r}=\beta\right\}, k^{\prime \prime}=$ $\left\{\beta \in k_{1}: \beta \pi^{r} \in K\left(x_{1}\right)\right\}$. Then $k^{\prime}$ and $k^{\prime \prime}$ have the same cardinality (Lemma 1 ), and $k^{\prime} \subseteq k^{\prime \prime}$, from the first part of this lemma. Hence $k^{\prime}=k^{\prime \prime}$.

Since we assume that $n$ is odd, we have $n_{0}=(n+1) / 2$. Let $H_{x}=G_{n_{0}} \cap(G \cap K(x))$; we write $H$ for $H_{x}$ when no confusion will result.

Lemma 3. $[H, H] \subseteq G_{n_{0}}$, and $\chi \equiv 1$ on $[H, H]$.

Proof. Because $K(x)$ is commutative and $G_{n_{0}}$ is normal, $[H, H] \subseteq$ $G_{n_{0}}$. For the rest, we need to show that if $w \in G_{n_{0}}$ and $y \in G \cap K(x)$, then $\chi\left(y w y^{-1}\right)=\chi(w)$. Let $w=1+w^{\prime}$; then

$$
\begin{aligned}
\chi\left(y w y^{-1}\right) & =\chi\left(1+y w^{\prime} y^{-1}\right) \\
& =\psi \circ \tau\left(y w^{\prime} y^{-1} x\right)=\psi \circ \tau\left(w^{\prime} y^{-1} x y\right) \\
& =\psi \circ \tau\left(w^{\prime} x\right)=\chi(w),
\end{aligned}
$$

since $x$ and $y$ commute.

There are $\left[H: G_{n}\right]$ different extensions of $\chi_{x}$ to $H$. Label them $\chi_{x ; a}$, where $a$ runs through the integers from 1 to $\left[H: G_{n_{0}}\right]$.

THEOREM 2. (a) $\rho_{x ; a}=\operatorname{Ind}_{H \rightarrow G} \chi_{x ; a}$ is irreducible.

(b) $\rho_{x ; a} \not \rho_{x ; b}$ unless $a=b$.

(c) The $\rho_{x ; a}$ exhaust the irreducible representations of $G$ containing $\chi$.

Proof. (a) It suffices, from Theorem 6 of [7], to show that if $y \notin H$, then there is some $w \in H$ such that $y w y^{-1} \in H$ and $\chi_{x ; a}(w) \neq$ $\chi_{x ; a}\left(y w y^{-1}\right)$. Let $y=1+\beta_{r} \pi^{r}+\cdots$; we may assume, since $y \notin H$ and we may multiply $y$ by any element of $H$, that $r<n_{0}$ and $\beta_{r} \pi^{r}$ does not commute with $x_{1}$ (see Lemma 2). Let $w=1+w^{\prime}$, with $w^{\prime} \in P^{n-r-2}$. Then

$$
\begin{aligned}
\chi_{x ; a}\left(y w y^{-1}\right) & =\psi \circ \tau\left(y w^{\prime} y^{-1} x\right) \\
& =\left(\psi \circ \tau\left(w^{\prime} x\right)\right)\left(\psi \circ \tau\left(w^{\prime} \beta_{r} \pi^{r} x-\beta_{r} \pi^{r} w^{\prime} x\right)\right),
\end{aligned}
$$

as one sees by expanding $y^{-1}=\left[1+\left(\beta_{r} \pi^{r}+\cdots\right)\right]^{-1}$ in a series. Therefore $\chi_{x ; a}\left(y w y^{-1}\right)=\chi_{x ; a}(w)$ for all such $w$ only if

$$
\begin{aligned}
1 & =\psi \circ \tau\left(w^{\prime} \beta_{r} \pi^{r} x-\beta_{r} \pi^{r} w^{\prime} x\right)=\psi \circ \tau\left(w^{\prime}\left(\beta_{r} \pi^{r} x-x \beta_{r} \pi^{r}\right)\right) \\
& =\psi \circ \tau\left(w^{\prime}\left(\beta_{r} \pi^{r} x_{1}-x_{1} \beta_{r} \pi^{r}\right)\right),
\end{aligned}
$$

since, e.g., $w^{\prime} \beta_{r} \pi^{r}\left(x-x_{1}\right) \in P^{-m-1}$, and so $\psi \circ \tau\left(w^{\prime} \beta_{r} \pi^{r}\left(x-x_{1}\right)\right)=1$. 
But since $w^{\prime}$ is any element of $P^{n-r-1}$, this implies that $\beta_{r} \pi^{r}$ and $x_{1}$ commute, a contradiction.

(b) If $\rho_{x ; a} \cong \rho_{x ; b}$ with $a \neq b$, then Theorem 7 of [7] implies that $\exists y \notin H$ such that for all $w \in H \cap y H y^{-1}, \chi_{x ; a}(w)=\chi_{x ; b}\left(y w y^{-1}\right)$. We saw in (a) that this is impossible.

(c) The dimension of $\operatorname{Ind}_{G_{n_{0} \rightarrow G}} \chi_{x}$ is [G: $\left.G_{n_{0}}\right]$ and the dimension of each $\rho_{x ; a}[G: H]$. As there are $\left[H: G_{n_{0}}\right]$ possible $\chi_{x ; a}$ 's, and as every irreducible $\rho$ containing $\chi_{x}$ occurs in $\operatorname{Ind}_{G_{n_{0}} \rightarrow G_{n}} \chi_{x}$ (by Frobenius reciprocity), the result follows from counting.

Next, we deal with Case (3). We continue with the notation as above. Let $H=G_{n_{0}}\left(G \cap D_{0}\right)$, where $D_{0}$ is the algebra defined in $\S 1$.

THEOREM 3. Suppose that $K\left(x_{1}\right)$ is unramified, with $\left[K\left(x_{1}\right): K\right]=p$. Then $D_{x_{1}}=D_{0}$. Let $\rho_{0}$ be any representation of $G \cap D_{0}$ such that $\left.\rho_{0}\right|_{a_{n-1} \cap D_{0}}$ is a multiple of $\left.\chi_{x}\right|_{a_{n-1} \cap D_{0}}$. Extend $\rho_{0}$ to a representation of letting $\rho_{0}\left(1+\alpha \pi^{r}\right)=I$ if $p \nmid r, r \geqq n_{0}$, and $\alpha \in k_{0}$. Then $\rho=$ $\operatorname{Ind}_{H \rightarrow G} \rho_{0}$ is irreducible, and $\left.\rho\right|_{G_{n-1}}$ is a multiple of $\left.\chi_{x}\right|_{G_{n-1}}$. If $\rho_{0}^{\prime}$ is another representation of $D_{0} \cap G$ satisfying the above condition and $\rho^{\prime}$ is the induced representation of $G$, then $\rho \cong \rho^{\prime} \Leftrightarrow \rho_{0} \cong \rho_{0}^{\prime}$. Every irreducible representation of $G$ which agrees with $\chi$ on $G_{n-1}$ is obtained in this way.

Proof. Since $K\left(x_{1}\right)$ is unramified, we have $\left.p^{2}\right|_{-n-m}$, and $K\left(x_{1}\right)=$ $K\left(\alpha_{-m-n}\right)=K_{1}$; thus $K\left(x_{1}\right)=K_{0}$, and $D_{x_{1}}=D_{0}$. Now the proof is the same as that of Theorem 2.3 of [1], except that, since $D_{0}=\{y=$ $\sum_{j=r}^{\infty} \beta_{j} \pi^{j}: \beta_{j}=0$ unless $\left.p \nmid j\right\}$, one can simplify the notation and some of the arguments.

REMARK. The above theorem does not directly identify the representations $\rho$ containing $\chi_{x}$. The following observations let one make this identification. For definiteness, assume that $[K(x): K]=p^{2}$. From Lemma 1, $K(x)$ contains a field isomorphic with $K\left(x_{1}\right)$; the Skolem-Noether theorem (see, e.g., p. 166 of [8]) lets us assume (by conjugating $x$ ) that $K\left(x_{1}\right) \leqq K(x)$. Then $\chi_{x}$ defines a character on $G_{n_{0}} \cap D_{0}$ which agrees with the norm map on $G_{n-1} \cap D_{0}$. As described in $\S$ IV of [4], we may use an appropriate extension of $\chi_{x}$ on a subgroup of $G \cap D_{0}$ to induce $\rho_{0}$ on $G \cap D_{0}$, and $\rho_{0}$ determines $\rho$. It is not hard to use this method to give an explicit description of the subgroup $H_{x}$ of $G$ such that $\rho$ is induced by $\chi$ extended to $H_{x}$. In the next section, we shall see more complicated examples along the same lines.

We should perhaps add a remark on the generality of these results. Theorem 2 applies to general $m$ (not just $m=p^{2}$ ); it thus permits the construction of many representations of $G$. So does 
Theorem 1, but that theorem reduces the problem only trivially. Theorem 3 permits a reduction of the problem whenever $\left[K\left(x_{1}\right): K\right]$ is unramified, but one must find a general procedure for finding the irreducible representations of $\left(D_{x_{1}}\right)^{x}$ to reap maximum benefit from this procedure.

3. We have reduced the problem to Case $(4): K\left(x_{1}\right)$ is a totally ramified extension of degree $p$. Thus $x_{1}^{p} \in K$. Because the map $\alpha \mapsto \alpha^{p}$ is an automorphism of $k$, one can see easily that there is an element $\beta \pi^{-m-n}$ with $\beta \in k_{1} \cap K$ and $x_{1}^{p}=\left(\beta \pi^{-m-n}\right)^{p}$. By conjugating with an element of $D$ (or, equivalently, by replacing $\pi$ with another prime element $\gamma \pi, \gamma \in k_{1}^{\prime}$, we may assume that $x_{1}=\beta \pi^{-m-n}$, or that $\alpha_{-m-n} \in K \cap k_{1}$. We keep to this assumption throughout this section and the next.

We divide Case 4 into three subcases, and we deal with the easiest one first.

LEMMA 4 . Let $x$ generate a field of degree $p$; let $y=\sum_{j=r}^{\infty} \beta_{j} \pi^{j}$ commute with $x$. Then $\beta_{r} \pi^{r}$ commutes with $x_{1}$. Conversely, if $\beta_{r} \pi^{r}$ commutes with $x_{1}$, then there is an element $y=\sum_{j=r}^{\infty} \beta_{j} \pi^{j}$ which commutes with $x$.

Proof. This has the same proof as in Lemma 2, once one notes that the division algebras $D_{x}$ and $D_{x_{1}}$ are both $p^{3}$-dimensional vector spaces over $K$, and that both $K_{x}, K_{x_{1}}$ are totally ramified of degree $p$ over $K$.

THEOREM 4. Let $x, x_{1}$ generate fields of degree $p$. Then:

(1) $\left.\chi_{x}\right|_{D_{x} \cap G_{n_{0}}}$ factors through the reduced norm $\nu_{D_{x} / K(x)}$;

(2) if $y \in D_{x} \cap G$ and $z \in G_{n_{0}}$, then $\chi_{x}\left(y z y^{-1}\right)=\chi_{x}(y)$.

Thus one can extend $\chi_{x}$ to a 1-dimensional representation of $G_{n_{0}}\left(G \cap D_{1}\right)$, also to be called $\chi_{x}$, which factors through the reduced norm on $G \cap D_{1}$. Let $\xi$ be any irreducible representation of $G \cap D_{1}$ which is trivial on $G_{n_{0}} \cap D_{1}$. Then $\xi \otimes \chi_{x}$ is a representation of $G_{n_{0}}\left(G \cap D_{1}\right)$ which is equivalent to a multiple of $\chi_{x}$ on $G_{n_{0}}$. Let $\rho_{\xi, x}=\operatorname{Ind}_{G_{n_{0}}\left(G \cap D_{1}\right) \rightarrow G} \xi \otimes \chi_{x}$. Then:

(3) $\rho_{\xi, x}$ is an irreducible representation of $G$;

(4) $\rho_{\xi_{1}, x} \cong \rho_{\xi_{2}, x} \Leftrightarrow \xi_{1} \cong \xi_{2}$;

(5) every irreducible representation of $G$ whose restriction to $G_{n_{0}}$ contains $\chi_{x}$ is a $\rho_{\xi, x}$.

Proof. (1) If $y=1+y^{\prime} \in D_{1} \cap G_{n_{0}}$, then let $x y^{\prime}$ generate a field $K^{\prime}$ over $K$. We have (assuming that $\left[K^{\prime}: K\right]=p^{2}$ )

$$
\begin{aligned}
\chi_{x}(y) & =\psi \circ \tau_{D / K}\left(x y^{\prime}\right)=\psi\left(T r_{K^{\prime} / K}(x y)\right)=\psi\left(T r_{K_{1} / K}\left(T r_{K^{\prime} / K}(x y)\right)\right) \\
& =\psi\left(T r_{K_{1} / K}\left(x T r_{K^{\prime} / K_{1}} y\right)\right)=\psi\left(T r_{K_{1} / K}\left(x \tau_{D_{1} / K_{1}} y^{\prime}\right)\right)
\end{aligned}
$$


This result also holds if $y^{\prime} \in K_{1}$. But $\nu_{D_{1^{\prime}} K_{1}}(y) \equiv 1+\tau_{D_{1^{\prime}} K_{1}} y^{\prime} \bmod P^{n}$, and this shows that $\chi_{x}(y)$ depends only on $\nu_{D_{1} / K_{1}}(y)$ for $y \in D_{1} \cap G_{n_{0}}$.

(2) If $z=1+z^{\prime} \in G \cap D_{1}$, then (for $y$ as above)

$$
\chi_{x}\left(y z y^{-1}\right)=\psi \circ \tau\left(y z^{\prime} y^{-1} x\right)=\psi \circ \tau\left(z^{\prime} y^{-1} x y\right)=\psi \circ \tau\left(z^{\prime} x\right)=\chi_{x}(z) .
$$

(3)-(5) The proof is just like that of Theorem 1, except that the counting argument is different. It is still routine; we omit details.

We are thus in the situation where $[K(x): K]=p^{2}$ and $K\left(x_{1}\right)$ is a totally ramified extension of degree $p$. This is the difficult case. We shall divide it into two subcases, depending on whether or not $K(x)$ is totally ramified. While the approach is similar in the subcases, and some lemmas could be combined, it seems clearer to divide the work.

If $K(x)$ contained a totally ramified extension (over $K$ ) of degree $p$, we could proceed just as in the tamely ramified case. In general, no such extension exists, and we must work harder.

For each $r$ with $1 \leqq r \leqq n_{0}$, let $x_{r}$ be an element of $D$ such that

(1) $x_{r} \equiv x \bmod P^{-m-n+r}$;

(2) $\left[K\left(x_{r}\right): K\right]$ is as small as possible, subject to (1). Note that this notation is consistent with the definition of $x_{1}$.

There is a number $s$ such that $\left[K\left(x_{s}\right): K\right]=p$ and $\left[K\left(x_{s+1}\right): K\right]=$ $p^{2}$. The general strategy of the proof is to show that for many purposes, we can use $K\left(x_{s}\right)$ as a substitute for a subfield of $K(x)$ of degree $p$ over $K$.

We begin by investigating some properties of $x_{8}$.

LEMMA 5. Let $y=\sum_{j=r}^{\infty} B_{j} \pi^{j} \in D$ generate a field of ramification index $p$ over $K$. Then there is a conjugate (under $G$ ) of $y, \sum_{j=r}^{\infty} \beta_{j} \pi^{j}$, with $\beta_{j}=0$ unless $p \mid j$.

Proof. $\quad D_{x}$ certainly contains an unramified extension $K_{0}^{\prime}$ of $K(y)$ with $\left[K_{0}^{\prime}: K(y)\right]=p$; by the Skolem-Noether theorem, we may assume (by conjugating in $D$ ) that $K_{0}^{\prime}=K(y, \alpha)$, where $K(\alpha)=K_{0}$. But under this assumption, $y$ commutes with $\alpha$, and this implies directly that the $\beta_{j}$ are 0 unless $p \mid j$. Since conjugating by an element of $k_{1}^{\prime}$ or by a power of $\pi$ gives an automorphism of $K_{0}$, we may equally well assume that the conjugation is in $G$.

LEMMA 6. Let $y=\sum_{j=r}^{\infty} \beta_{j} \pi^{j}$ generate a totally ramified extension of order $p$; let $y_{1}=\beta_{r} \pi^{\tau}$. Assume that $\beta_{j}=0$ unless $p \mid j$ and that $\left[K\left(y_{1}\right): K\right]=p, \beta_{r} \in k$. Let $w=\beta^{\prime} \pi^{t}, t>r$, be such that if $y^{\prime} \equiv y+w$ $\left(\bmod P^{t+1}\right)$, then $\left[K\left(y^{\prime}\right): K\right]=p^{2}$, and assume further that $K(y+w)$ is 
not totally ramified over $K . \quad$ (Therefore $f(K(y+w) / K)=p$.) Then:

(a) $p \mid t$ and $T r_{d / k_{0}} \beta^{\prime} \notin k$;

(b) if $y^{\prime} \equiv y+w \bmod P^{t+1}$, then $f\left(K\left(y^{\prime}\right) / K\right)=p$.

Proof. Note that $K_{0} \subseteq D_{y}$. Since multiplying by an element of $K$ does not affect the hypotheses, we may assume that $0<r<p^{2}$. Let $F$ be the minimal polynomial for $y$ :

$$
F(X)=\sum_{j=0}^{p} a_{j} X^{j}, \quad a_{p}=1 \text { and } a_{j} \in \mathfrak{O}_{K} \text {, all } j .
$$

By considering the valuation (plus the fact that $F(y)=0$ ), one sees that $a_{0} \in P^{p r} / P^{p r+1}$ and that the terms $a_{j} y^{j}(1 \leqq j \leqq p-1)$ are in $p^{p(r+1)}$. (Note that $p \mid r$.)

Now consider $F(y+w)$; again, valuation considerations show that

$$
F(y+w) \equiv x^{p-1}\left(T r_{d / k_{0}} \beta^{\prime}\right) \pi^{t} \bmod P^{(p-1) r+t+1} .
$$

If $p \nmid t$ and $T r_{d / k_{0}} \beta^{\prime} \neq 0$, then this last calculation shows that $F(y+w)$ is an element which generates an ideal $P^{t_{0}}$ with $\left(t_{0}, p\right)=1$; it follows that $K(y+w)$ is totally ramified. On the other hand, if $T r_{d / k_{y}} \beta^{\prime}=0$, then $\beta^{\prime}$ is of the form $\gamma^{\sigma^{r}}-\gamma$ for some $\gamma \in d_{0}$ (where $p \mid r$ and $p^{2} \nmid r$ ), and

$$
\left(1-\gamma \pi^{t-r}\right) y\left(1-\gamma \pi^{t-r}\right)^{-1} \equiv y+w \bmod P^{t+1} .
$$

Thus there is an element $y^{\prime} \equiv y+w \bmod P^{t+1}$ with $\left[K\left(y^{\prime}\right): K\right]=p$, a contradiction. It follows that $p \mid t$.

We know from general structure theory (and the fact that $K_{0} K(y)$ is a maximal unramified field in $D_{y}$ ) that $\exists u \in D_{y}$ such that $u^{p}=y$ and conjugation by $u$ induces an automorphism generating Gal $\left(K_{0} / K\right)$. Then $u=\sum_{j=s}^{\infty} \delta_{j} \pi^{j}$, where $p s=r, \delta_{s} \in k_{1} \cap K, \delta_{s}^{p}=\beta_{r}$, and $\delta_{j}=0$ unless $j \equiv s \bmod p$. (This last follows because otherwise conjugating by $u$ will not be an automorphism of $K_{0}$.) Because $\beta_{r} \in k$ and taking $p$ th powers is an automorphism of $k, \delta_{s} \in k$. Let $u^{\prime}=$ $u+\gamma_{0} \pi^{s+(t-r)}$; note that $p \mid t-r$. Then

$$
\left(u^{\prime}\right)^{p} \equiv y+\delta_{s}\left(\gamma_{0}+\gamma_{0}^{\sigma^{s}}+\cdots+\gamma_{0}^{\sigma^{(p-1) s}}\right) \pi^{t} \bmod P^{t+1} .
$$

So if $\beta^{\prime}$ can be written as $\beta_{s}\left(\gamma_{0}+\gamma_{0}^{\sigma^{s}}+\cdots+\gamma_{0}^{\left.\sigma^{(p-1)}\right)}\right)$, then there exists $y^{\prime} \equiv y+w \bmod P^{t+1}$ such that $y^{\prime}$ is a $p$ th power in $D$; then, of course, $\left[K\left(y^{\prime}\right): K\right]<p^{2}$, and we again have a contradiction. Thus $\beta^{\prime}$ is not of that form.

Let $\sigma^{\prime}=\sigma^{s}$; let $T \gamma=\gamma+\gamma^{\sigma^{\prime}}+\cdots+\gamma^{\left(\sigma^{\prime}\right) p-1}, \gamma \in k_{1}$. Note that $T$ is $k$-linear and $\delta_{s} \in k$, so that $\beta^{\prime} \notin \operatorname{Range}(T)$. Thus we need to show that Range $T=\left\{\delta \in d: T r_{d / k_{0}} \delta \in k\right\}$. Call this last described set $V$. Then $V$ is a vector space over $k$ of dimension $p^{2}-p+1$, since 
$\operatorname{Ker}\left(T r_{d / k_{0}}\right)$ is a $(p-1)$-dimensional $k_{0}$-space. On the other hand, since $\sigma^{\prime}$ generates $\operatorname{Gal}(d / k), T \delta \in k \Leftrightarrow \sigma^{\prime}(T \delta)=T \delta \Leftrightarrow \delta=\delta^{\left(\sigma^{\prime}\right) p} \Leftrightarrow \delta \in k_{0}$, and on $k_{0}, T=T r_{k_{0} / k}$. Hence

$$
\operatorname{Ker} T=\operatorname{Ker}\left(T r_{k_{0} / k}\right),
$$

a $(p-1)$-dimensional $k$-subspace of $k_{0}$. Thus Range $T$ and $V$ have the same dimension. Since, however, $T r_{d / k_{0}} \circ T=T r_{d / k}$, we have $\gamma \in$ Range $T \Rightarrow T r_{d / k_{0}} \gamma \in k$, or Range $T \subseteq V$. This proves (a).

For (b), note that if $y^{\prime} \equiv y+w \bmod \pi^{t}$, then $F\left(y^{\prime}\right) \equiv x^{p-1}\left(T r_{d / k_{0}} \beta\right) \pi^{t}$ $\bmod P^{(p-1) r+t+1}$; thus $\alpha\left(y^{\prime}\right)^{a} F\left(y^{\prime}\right)\left(\pi^{\rho^{2}}\right)^{b}=T r_{d / k_{0}} \beta \bmod P$ for appropriate $\alpha \in k_{0}$ and $a, b \in Z$. By Hensel's lemma, $K\left(y^{\prime}\right)$ contains an unramified extension of order $p$.

Now we return to the problem of representations. We let $\chi=$ $\chi_{x}$, where $[K(x): K]=p^{2},\left[K\left(x_{1}\right): K\right]=p, K\left(x_{1}\right)$ is totally ramified over $K$, and $K(x)$ is not totally ramified. We choose $x_{s}$ as in the discussion before Lemma 5 . Then $x$ satisfies the hypotheses of Lemma 5, and we may thus assume that $x=\sum_{j=m-n}^{\infty} \alpha_{j} \pi^{j}$, with $\alpha_{j}=0$ when $p \nmid j$. Moreover, $x_{s}$ and $w=x-x_{s}$ satisfy the hypotheses of Lemma 6. Note that $p \mid s$.

We need to show that certain elements of $D_{x_{s}}$ are "close" to elements of $K(x)$, so that we will be able to use $K\left(x_{s}\right)$ as if it were a subfield of $K(x)$. The next lemma gives what is needed.

LEMma 7. We can find elements $t^{\prime}, t$ and $u$ such that:

(a) $t^{\prime}$ is a prime element of $K(x)$, and $u$ is a prime element of $D_{x_{8}}$;

(b) $u$ commutes with $t^{\prime} \bmod P^{p+s+1}$; that is, $u t^{\prime}-t^{\prime} u \in P^{p+s+1}$;

(c) $u^{p}=t$, and $t$ is a prime element of $K\left(x_{s}\right)$ with $t^{\prime} \equiv t$ $\bmod P^{p+s}$.

Proof. Recall that $x=\alpha_{j p} \pi^{j p}+$ higher order terms, where $j p=$ $-m-n$. Choose $h$ so that $j h \equiv 1 \bmod p$; then we may find $a \in K$ such that $t^{\prime}=a x^{h}$ generates $P^{p}$. In fact, we may assume that $t^{\prime}=$ $\pi^{p}+$ higher order terms. Let $t=a x_{x}^{h}$. Then $t^{\prime} \equiv t \bmod P^{p+s}$. Since $t$ generates a field of degree $p$ over $K$, we can find a $p$ th root $u$ of $t$ in $D$ with $u=\sum_{j=1}^{\infty} \beta_{j} \pi^{j}, 0 \neq \beta_{1} \in k$, as was done in Lemma 6 . Then, of course, $u$ commutes with $t^{\prime} \bmod P^{p+s+1}$, since $u \in P$ and $t^{\prime}-t \in P^{p+s}$.

Now assume for simplicity that $s$ is even; let $s=2 s_{0}$. We define

$$
H_{x}=G_{n_{0}}\left(D_{x_{s}} \cap G_{n_{0}-s_{0}}\right)(G \cap K(x)) \text {. }
$$

Note that coset representatives for $D_{x_{s}} \cap\left(1+P^{j}\right) / D_{x_{s}} \cap\left(1+P^{j+1}\right)$ are 
given by elements $1+\gamma u^{r}, \gamma \in k_{0}$ and $u$ as in Lemma 7 , and that coset representatives for $K(x) \cap\left(1+P^{j p}\right) / K(x) \cap\left(1+P^{j p+1}\right)$ are given by elements $1+\gamma\left(t^{\prime}\right)^{j}, \gamma \in d_{0}$. (If $p \nmid r, K(x) \cap\left(1+P^{r}\right)=K(x) \cap\left(1+P^{r+1}\right)$.)

The next lemma is the crucial one for extending $\chi$ to $H_{x}$.

Lemma 8. (a) If $n_{0}-s_{0}<j<n_{0}$, then

$$
\begin{aligned}
{\left[H_{x}, H_{x}\right] \cap\left(1+P^{j}\right) /\left[H_{x}, H_{x}\right] \cap\left(1+P^{j+1}\right) } \\
\cong \begin{cases}\left(1+P^{j}\right) /\left(1+P^{j+1}\right) & \text { if } p \nmid j \text { and there is a multiple } \\
\{1\} & \text { of } p \text { between } n_{0}-s_{0} \text { and } j ;\end{cases}
\end{aligned}
$$

(b) If $z \in\left[H_{x}, H_{x}\right] \cap\left(1+P^{n_{0}}\right)$, then $\chi_{x}(z)=1$.

REMARK. Part (b) of the lemma says that $\chi_{x}$ has an extension to $H_{x}$. Any such extension must be trivial on $\left[H_{x}, H_{x}\right]$, of course; part (a) therefore enables us to count the extensions of $\chi_{x}$.

Proof. We use the notation of Lemma 7.

(a) Modulo $G_{n_{0}}$, the commutators generating $\left[H_{x}, H_{x}\right]$ are of the form

$$
(f, g)^{\prime}=\left(1+f\left(t^{\prime}\right)\right)(1+g(u))\left(1+f\left(t^{\prime}\right)\right)^{-1}(1+g(u))^{-1},
$$

where $f$ is a polynomial over $K_{0}$ with integral coefficients and no constant term, while $g$ is a polynomial with integral coefficients in $K_{0}$ and all terms of degree $\geqq n_{0}-s_{0}$. (The coefficients of $g(u)$ need not commute with $u$.) Substitute

$$
\begin{aligned}
& \left(1+f\left(t^{\prime}\right)\right)^{-1}=1-f\left(t^{\prime}\right)+f\left(t^{\prime}\right)^{2}+\cdots, \\
& (1+g(u))^{-1}=1-g(u)+g(u)^{2}+\cdots,
\end{aligned}
$$

in the formula for $(f, g)^{\prime}$. Since $g(u)^{2} \in P^{n_{0}}$ and we are calculating modulo $P^{n_{0}}$, we can drop all powers of $g(u)$ beyond the first, setting

$$
\begin{aligned}
(f, g)^{\prime} & \equiv 1+\sum_{i=0}^{n_{0}}(-1)^{i}\left[f\left(t^{\prime}\right)^{i+1} g(u)-f\left(t^{\prime}\right) g(u) f\left(t^{\prime}\right)^{i}\right] \\
& \equiv 1+\sum_{i=0}^{n_{0}}(-1)^{i}\left[f(t)^{i+1} g(u)-f(t) g(u) f(t)^{i}\right] .
\end{aligned}
$$

This last congruence holds because $f(t) \equiv f\left(t^{\prime}\right) \bmod P^{p+s}$ and $g(u) \in P^{n_{0}-s_{0}}$.

Any term in $g(u)$ whose degree is a multiple of $p$ can be written as a power of $t$, since $t=u^{p}$; that term commutes with $f$. Let $g_{0}$ be the polynomial obtained from $g$ by omitting all terms whose degree is a multiple of $p$. Then

$$
\left(f, g^{\prime}\right) \equiv 1+\sum_{i=0}^{n_{0}}(-1)^{i}\left[f\left(u^{p}\right)^{i+1} g_{0}(u)-f\left(u^{p}\right) g(u) f\left(u^{p}\right)^{i}\right],
$$


and every term in this expression is of degree prime to $p$ and greater than $n_{0}-s_{0}$. To complete the proof of (a), note that if $n_{0}-s_{0} \leqq$ $j_{0} p<j_{0} p+j_{1}=j<n_{0}$, then the elements

$$
\left(1+\left(\beta t^{\prime}\right)^{j_{0}}\right)\left(1+\gamma u^{j_{1}}\right)\left(1+\left(\beta t^{\prime}\right)^{j_{0}}\right)^{-1}\left(1+\left(\gamma u^{j_{1}}\right)\right)^{-1},
$$

where $\beta, \gamma$ vary over $k_{0}$, give the coset representatives.

(b) This is more complicated; the idea is to replace computations of $\chi_{x}$ with computations of $\chi_{x_{s}}$. Define $(f, g)^{\prime}$ as in (a), and set

$$
(f, g)_{1}^{\prime}=\sum_{i=0}^{\infty}(-1)^{i}\left[f\left(t^{\prime}\right)^{i+1} g(u)-f\left(t^{\prime}\right) g(u) f\left(t^{\prime}\right)^{i}\right] .
$$

If $w^{\prime}=\prod_{j=1}^{h}\left(f_{j}, g_{j}\right)^{\prime}$, let $w_{1}^{\prime}=\sum_{j=1}^{h}\left(f_{j}, g_{j}\right)_{1}^{\prime}, w_{2}^{\prime}=w^{\prime}\left(1+w_{1}^{\prime}\right)^{-1}$. One can think of $w_{1}^{\prime}$ as containing all the terms in $w^{\prime}$ that are linear in some $g_{j}$. Similarly, we define

$$
\begin{aligned}
(f, g) & =(1+f(t))(1+g(u))(1+f(t))^{-1}+(1+g(u))^{-1}, \\
(f, g)_{1} & =\sum_{i=0}^{\infty}(-1)^{i}\left[f(t)^{i+1} g(u)-f(t) g(u) f(t)^{i}\right] ;
\end{aligned}
$$

note that we have replaced $t^{\prime}$ with $t$. If $w^{\prime}$ is as above, let

$$
w=\prod_{j=1}^{h}\left(f_{j}, g_{j}\right), \quad w_{1}=\sum_{j=1}^{h}\left(f_{j}, g_{j}\right)_{1}, \quad w_{2}=w\left(1+w_{1}\right)^{-1} .
$$

Now:

(1) $w \in G_{n_{0}}$. For $w^{\prime} \in G_{n_{0}}$, and (as we saw in the proof of part (a)) replacing $t^{\prime}$ by $t$ in the commutators changes terms only by elements of $P^{n_{0}}$.

(2) $w_{2}^{\prime} \equiv w_{2} \bmod G_{n+1}$. For $w_{2}^{\prime}-1$ is a sum of terms involving products of two or more $g_{j}(u)$ and one or more $f_{i}\left(t^{\prime}\right)$, and $w_{2}-1$ can be written as a similar sum involving the $g_{j}(u)$ and $f_{i}(t)$. Since $g_{j_{1}}(u) g_{j_{2}}(u) \in P^{n-s+1}$ and $t^{\prime}-t \in P^{s}$, we have $w_{2}^{\prime}-w_{2} \in P^{n+1}$.

(3) $w_{2}^{\prime} \in G_{n-s+1}$. This follows from the calculation in (2), since $g_{j_{1}}(u) g_{j_{2}}(u) \in G_{n-8+1}$.

(4) $\chi_{x}\left(w_{2}^{\prime}\right)=\chi_{x_{s}}\left(w_{2}^{\prime}\right)=\chi_{x_{s}}\left(w_{2}\right)$, from (2) and (3), since $\chi_{x}$ and $\chi_{x_{s}}$ agree on $G_{n-8+1}$.

(5) $\chi_{x}\left(1+w_{1}^{\prime}\right)=1$. For

$$
\chi_{x}\left(1+w_{1}^{\prime}\right)=\psi\left(\tau_{D / K}\left(w_{1}^{\prime} x\right)\right),
$$

and, because $x$ and $t^{\prime}$ commute,

$$
\begin{aligned}
\tau\left(w_{1}^{\prime} x\right) & =\tau\left(\sum_{j=1}^{h} \sum_{i=0}^{\infty}(-1)^{i}\left(f_{j}\left(t^{\prime}\right)^{i+1} g_{j}(u) x-f_{j}\left(t^{\prime}\right) g_{j}(u) f_{j}\left(t^{\prime}\right)^{i} x\right)\right) \\
& =\tau\left(\sum_{j=1}^{h} \sum_{i=0}^{\infty}(-1)^{i}\left(g_{j}(u) x f_{j}\left(t^{\prime}\right)^{i+1}-g(u) f_{j}\left(t^{\prime}\right)^{i} x f_{j}\left(t^{\prime}\right)\right)\right) \\
& =0 .
\end{aligned}
$$


(6) $\chi_{x_{s}}\left(1+w_{1}\right)=1$, by a calculation like that in (5), since $x_{s}$ and $t$ commute.

(7) $\chi_{x}\left(w^{\prime}\right)=\chi_{x_{s}}(w)$, from (4), (5) and (6).

(8) Thus we need only compute $\chi_{s}(w)$. But $w$ is a commutator in $D_{x_{s}} \cap\left(1+P^{n_{0}}\right)$, and therefore $w=1+w_{0}$, with $\tau_{D_{x_{s} / R\left(x_{s}\right)}} w_{0} \equiv 0 \bmod P^{n+1}$. Hence $\tau\left(w_{0} x_{s}\right) \equiv 0 \bmod P^{n+1}$, and hence $\chi_{x_{s}}\left(w_{0}\right)=1$.

To finish the proof of (b), we need only mention that all commutators in $H_{x} \cap G_{n_{0}}$ are products of the commutators just considered with commutators of the form $z=z_{1} z_{2} z_{1}^{-1} z_{2}^{-1}, z_{1}, z_{2} \in D_{x_{s}} \cap G_{n_{0}-8_{0}}$. Then $z \in G_{n-s+1}$, and so $\chi_{x}(z)=\chi_{x_{s}}(z)=1$ for the same reasons as in (4) and (8) above.

We label the different extensions of $\chi_{x}$ to $H_{x}$ with integers; a typical one is $\chi_{x, a}$. Let $p j_{1}$ be the smallest multiple of $p$ that is greater than $n_{0}-s_{0}$. We say that $\chi_{x, a}$ and $\chi_{x, b}$ are equivalent if they agree on $K(x) \cap H_{x}$. (Thus they may differ on elements $1+\eta u^{r}$, $n_{0}-s_{0} \leqq r<p j$.)

It will be convenient in what follows to have a reference additive character $\psi^{(s)}$ defined on $K\left(x_{s}\right)$ which corresponds to $\psi$ on $K$. (Recall: $\psi$ is an additive character. See $\S 1$.) We let $\psi^{(s)}$ be any character on $K\left(x_{s}\right)$, trivial on $P^{-m+p}$, whose restriction to $K$ is $\psi$. Note that if $\psi_{k}$ is the character defined on $k$ by $\psi_{k}(\gamma)=\psi\left(\gamma \pi^{-m}\right)$, then $\psi^{(s)}\left(\gamma t^{-p}\right)=\psi_{k}(\gamma), \forall \gamma \in k$.

TheOREM 5. (a) If $\chi_{x ; a}$ is any extension of $\chi_{x}$ to $H_{x}$, then $\operatorname{Ind}_{H_{x \rightarrow G} \rightarrow} \chi_{x ; a}=\rho_{x ; a}$ is irreducible.

(b) Inequivalent $\chi_{x ; a}$ give rise to inequivalent $\rho_{x ; a}$, and equivalent $\chi_{x ; a}$ give rise to equivalent $\rho_{x ; a}$.

(c) Any irreducible representation of $G$ whose restriction to $G_{n_{0}}$ contains $\chi_{x}$ is equivalent to some $\rho_{x ; a}$.

Proof. (a) We need to show that if $z=1+\sum_{j=r}^{\infty} \gamma_{j} \pi^{j}, r \geqq 1$, is such that $\chi_{x ; a}\left(z y z^{-1}\right)=\chi_{x ; a}(z)$ whenever $y, z y z^{-1}$ are both in $H_{x}$, then $z \in H_{x}$. Suppose otherwise. We may then assume that $z z^{\prime} \notin G_{r+1}$ whenever $z^{\prime} \in H_{x}$.

Let $y=1+y_{0}, y_{0} \in P^{n_{0}}$. Then $\chi_{x ; a}(y)=\chi_{x}(y)$ and $\chi_{x ; a}\left(z y z^{-1}\right)=$ $\chi_{x}\left(z y z^{-1}\right)=\chi_{z^{-1} x z}(y)$, so that $z^{-1} x z \equiv z \bmod P^{-n_{0}-m}$. In particular, $\gamma_{r} \pi_{r}$ commutes with $x_{1}$. From Lemma 4 (applied to $x_{s}$ ), there is an element $z_{0}=1+\gamma_{r} \pi^{r}+$ higher order terms with $z \in D_{x_{s}}$. As $z z_{0}^{-1} \in G_{r+1}$, we may not have $z_{0} \in H_{x}$. Therefore $r<n_{0}-s_{0}$. Moreover, if $p \mid r$, then there is an element $q^{\prime}=1+\gamma_{r} \pi^{r}+$ higher order terms with $z^{\prime} \in K(x)$. We conclude that $p \nmid r$.

Thus far, we have dealt only with $\chi_{s}$; now we analyze $\chi_{x ; a}$ more carefully. On $D_{x_{s}} \cap G_{n_{0}}, \chi_{x_{s}}$ factors through $\nu_{D_{x_{s}} / K\left(x_{s}\right)}$. The proof of 
this fact parallels that of Theorem 1. Define the character $\psi_{0}$ on $K\left(x_{s}\right)$ by $\psi_{0}=\psi \circ T r_{K\left(x_{s}\right) / K}$. If $1+y \in D_{x_{s}} \cap G_{n_{0}}$, then

$$
\nu_{D_{x_{s} / K\left(x_{s}\right)}}(1+y) \equiv 1+\tau_{D_{x_{s} / K\left(x_{s}\right)} y}\left(\bmod P^{n+1}\right),
$$

and so $\chi_{x_{s}}(1+y)=\psi_{0}\left(x_{s} \tau_{D_{x_{s} / K\left(x_{s}\right)}} y\right)$ depends only on $\nu(y)$.

Let $\omega$ be a 1-dimensional representation of $D_{x_{s}} \cap G$ which agrees with $\chi_{x_{s}}$ on $D_{x_{s}} \cap G_{n_{0}}$. Then $\chi_{x} \omega^{-1}$ is trivial on $D_{x_{s}} \cap G_{n-s}$. On $D_{x_{s}} \cap$ $G_{n_{0}-s_{0}}$, we may write

where

$$
\chi_{x ; a} \omega^{-1}(1+y)=\psi^{(s)} \tau_{D_{x_{s} / K\left(x_{s}\right)}}\left(y y_{0}\right),
$$

$$
y_{0}=\sum_{j=-n+s-m}^{\infty} \beta_{j} \pi^{j} \in D_{x_{s}} .
$$

Let $y_{1}=\beta_{-n+s-m} u^{-n+s-m}$, and write $\delta=\beta_{-n+s-m}$; let $x-x_{s}=\gamma \pi^{-n+s-m}+$ higher order terms.

Since $x-x_{s}$ and $y_{1}$ both describe the behavior of $\chi_{s: a} \cdot \omega^{-1}$ on $G_{n-s}$, they should be related. We now describe the relationship. If $y=$ $1+\beta u^{n-s} \in D_{x_{s}}$, then

$$
\begin{aligned}
\chi_{x ; a} \circ \omega^{-1}(y) & =\chi_{x-x_{s}}(y)=\psi \circ \tau_{D / K}\left((y-1)\left(x-x_{s}\right)\right) \\
& =\psi \circ \tau_{D / K}\left(\beta \gamma^{\sigma^{n-s}} \pi^{-m}\right)=\psi \gamma_{k} \circ T r_{d / k}\left(\beta \gamma^{\sigma^{n-s}}\right) \\
& =\psi_{k} \circ T r_{d / k}\left(\gamma \beta^{\sigma^{s-n}}\right)=\psi_{k} \circ T r_{d_{0} / k} \beta^{\sigma^{s-n}}\left(T r_{d / d_{0}} \gamma\right)
\end{aligned}
$$

since $\beta \in d_{0}$. Also,

$$
\begin{aligned}
\chi_{x ; a} \cdot \omega^{-1}(y) & =\psi^{(s)} \circ \tau_{D_{x_{s}} / K\left(x_{s}\right.}\left((y-1) y_{0}\right) \\
& =\psi^{(s)} \circ \tau_{D_{x_{s} / K(x)}}\left(\beta \delta^{o n-s} \pi^{-m}\right) \\
& =\psi_{k} \circ T r_{d_{0} / k}\left(\beta \delta^{\sigma^{n-s}}\right)=\psi_{k} \circ T r_{d_{0} / k}\left(\beta^{\sigma^{s-n}} \delta\right) .
\end{aligned}
$$

Thus $\delta=T r_{d / d_{0}} \gamma$. By Lemma $6, \delta \notin k_{0}$. Therefore $y_{1}$ generates an unramified extension of order $p$ over $K\left(x_{s}\right)$.

We use this fact to restrict the element $z$ further. On $H_{x} \cap D_{x_{s}}$, conjugating by $z$ commutes with $\omega$. Therefore it commutes with $\chi_{x ; a} \cdot \omega^{-1}$. Let $y \in D_{x_{s}} \cap G_{n-s-r}$. Then

$$
\chi_{x ; a} \cdot \omega^{-1}(y)=\chi_{x ; a} \cdot \omega^{-1}\left(z y z^{-1}\right),
$$

and a calculation like that at the start of the proof shows that

$$
z^{-1} y_{0} z \equiv y_{0} \quad \bmod P^{-n_{0}-s_{0}-m} \text {. }
$$

In particular, $y_{1}$ and $\gamma_{r} \pi^{r}$ commute. But the residue class degree of $y_{1}$ is divisible by $p$; it follows that $p \mid r$. This contradicts our earlier demonstration that $p \nmid r$, and part (a) follows.

(b) If $\rho_{x ; a}$ and $\rho_{x ; b}$ are equivalent, then $\exists z: \chi_{x ; a}(y)=\chi_{x ; b}\left(z y z^{-1}\right)$ whenever $y$ and $z y z^{-1}$ are both in $H_{x}$. In particular, this holds for all $y \in G_{n_{0}}$. Write $z$ as in part (a). The analysis given there shows that 
$z \in D_{x_{s}} \cap G_{n_{0}-8}$, that $p \nmid r$, and that conjugation by $z$ changes $\chi_{x ; a}$ on elements of the form $1+\beta u^{n-s-r}$. We know that $p \nmid n-s-r$. If $n-s-r>p j_{0}$ (where $p j_{0}$ is the smallest multiple of $p$ greater than $n_{0}-s_{0}$ ), then $\chi_{x ; a}$ and $\chi_{x: b}$ must agree, from (a) of Lemma 8. Hence $n-s-r<p j_{0}$. It is now easy to check that $z$ normalizes $H_{x}$ and that $\chi_{x: a}$ and $\chi_{x: b}$ are equivalent.

That equivalent $\chi_{x ; a}$ produce equivalent $\rho_{x ; a}$ can be proved by picking $z$ as in the above paragraph. It is also implied by (c); see below.

(c) This is a matter of counting. Observe first that if $z \in G$ fixes $\chi_{x}$, then (modulo $H_{x}$ ) $z \in D_{x_{s}} \cap G_{n_{0}-8}$. (This follows from the analysis in part (a); the only difference is that in the second part of the analysis, we are concerned only with elements $y \in D_{x_{s}} \cap G_{n_{0}}$.) Conversely, a straightforward calculation shows that any such $z$ does fix $\chi_{x}$. Hence the multiplicity of $\chi_{x}$ in $\rho_{x ; a} \mid G_{n_{0}}$ is $\left[D_{x_{s}} \cap G_{n_{0}-s}: D_{x_{s}} \cap\right.$ $\left.G_{n_{0}-8_{0}}\right] /\left[K(x) \cap G_{n_{0}-8}: K(x) \cap G_{n_{0}-\varepsilon_{0}}\right]$. By Frobenius reciprocity, this is also the multiplicity of $\rho_{x ; a}$ in $\operatorname{Ind}_{a_{n_{0} \rightarrow G}} \chi_{x}$. We also know that there are $\left[G \cap K(x): G_{n_{0}} \cap K(x)\right.$ ] equivalence classes of $\chi_{x: a}$ 's and that

$\operatorname{Dim} \rho_{x: a}=\left[G: H_{x}\right]$

$$
=\left[G / G_{n_{0}}:(G \cap K(x))\left(G_{n_{0}-s_{0}} \cap D_{x_{8}}\right) /\left(G_{n_{0}} \cap K(x)\right)\left(G_{n_{0}} \cap D_{x_{s}}\right)\right] .
$$

Since $(G \cap K(x)) \cap\left(G_{n_{0}-s_{0}} \cap D_{x}\right)=G_{n_{0}-s_{0}} \cap K(x)$ and $\left(G_{n_{0}} \cap K(x)\right) \cap\left(G_{n_{0}} \cap\right.$ $D(x))=G_{n_{0}} \cap K(x)$, we have

$$
\begin{aligned}
\operatorname{Dim} \rho_{x ; a}= & {\left[G / G_{n_{0}}: G \cap K(x) / G_{n_{0}} \cap K(x)\right] } \\
& \times\left[G_{n_{0}-s_{0}} \cap D_{x_{s}} / G_{n_{0}} \cap D_{x_{s}}: G_{n_{0}-s_{0}} \cap K(x) / G_{n_{0}} \cap K(x)\right]^{-1} .
\end{aligned}
$$

Thus the different $\chi_{x ; a}$ account for a subspace in $\operatorname{Ind}_{G_{n_{0} \rightarrow G}} \chi_{x}$ of dimension

$$
\begin{aligned}
{\left[G / G_{n_{0}}\right]\left[G \cap K(x) / G_{n_{0}} \cap K(x)\right]^{-1}\left[G_{n_{0}-s_{0}} \cap D_{x_{s}} / G_{n_{0}} \cap D_{x_{s}}\right]^{-1} } \\
\quad \times\left[G_{n_{0}-s_{0}} \cap K(x) / G_{n_{0}} \cap K(x)\right]\left[G \cap K(x) / G_{n_{0}} \cap K(x)\right] \\
\quad \times\left[D_{x_{s}} \cap G_{n_{0}-s} / D_{x_{s}} \cap G_{n_{0}-s_{0}}\right]\left[K(x) \cap G_{n_{0}-s}: K(x) \cap G_{n_{0}-s_{0}}\right]^{-1} .
\end{aligned}
$$

But $\left[G_{n_{0}-s_{0}} \cap K(x) / G_{n_{0}} \cap K(x)\right] /\left[G_{n_{0}-8} \cap K(x) / G_{n_{0}-s_{0}} \cap K(x)\right]=1$ and $\left[D_{x_{s}} \cap G_{n_{0}-s_{0}} / D_{x_{s}} \cap G_{n_{s}}\right] /\left[D_{x_{s}} \cap G_{n_{0}-s} / D_{x_{s}} \cap G_{n_{0}-s_{0}}\right]=1$. (Note that $p \neq 2$, since $p \mid n$ and $n$ is odd; also, $p \mid s$. Hence $p \mid s_{0}$, and the number of multiples of $p$ between $n_{0}-s_{0}$ and $n_{0}$ equals the number between $n_{0}-s$ and $n_{0}-s_{0}$. From this, the first relation follows; the second is even easier to verify.) That is, the $\chi_{x ; a}$ account for a subspace in $\operatorname{Ind}_{G_{n_{0} \rightarrow G}} \chi_{x}$ of dimension $\left[G: G_{n_{0}}\right]=\operatorname{dim} \operatorname{Ind}_{G_{n_{0}} \rightarrow G} \chi_{x}$.

That proves (c). Also, as we have accounted for all the representations in $\operatorname{Ind}_{G_{n_{0} \rightarrow G}} \chi_{x}$, it must be the case that $\chi_{x ; a}$ equivalent to $\chi_{x, b}$ implies $\rho_{x ; a} \cong \rho_{x ; b}$.

4. We still need to deal with the subcase in which $\left[K\left(x_{1}\right): K\right]=p$, 
$[K(x): K]=p^{2}$, and $K(x)$ is totally ramified. This situation is similar to the last one we dealt with, but there are complications.

We define $x_{s}$ as in the previous section. We begin with three lemmas similar to Lemmas 6,7 and 8 .

Lemma 9. Let $y$ be as in Lemma 6. Let $w=\beta \pi^{t}, t>r$, be such that if $y^{\prime} \equiv y+w \bmod P^{t+1}$, then $\left[K\left(y^{\prime}\right): K\right]=p^{2}$, and suppose that $K(y+w)$ is totally ramified over $K$. Then:

(a) $p \nmid t$ and $T r_{d / k_{0}} \beta \neq 0$;

(b) if $y^{\prime} \equiv y+w \bmod P^{t+1}$, then $K\left(y^{\prime}\right)$ is totally ramified over $K$;

(c) by perhaps changing the choice of prime element, one can also arrange that $\operatorname{Tr}_{d / k_{0}} \beta \in k$ (without upsetting the other normalizations already made).

Proof. The proofs of (a) and (b) are like that of Lemma 6, with easy modifications. For (c), notice first that if $\delta \in k_{0}$, then $(\delta \pi)^{p}=\delta^{\prime} \pi^{p}$, $\delta^{\prime} \in k$. Thus using $\pi^{\prime}=\delta^{-1} \pi$ as prime element does not affect our assumptions about $y$. On the other hand, this change means that $w=\varepsilon \beta\left(\pi^{\prime}\right)^{t}$, where $\varepsilon=\delta \delta^{\sigma} \cdots\left(\delta^{\sigma}\right)^{t-1}$; of course, $T r_{d / k_{0}} \varepsilon \beta=\varepsilon T r_{d / k_{0}} \beta$, and $N_{k_{0} / k} \varepsilon=(N \delta)^{t}$. Because $d^{x}$ is cyclic, we can modify $T r_{d / k_{0}} \beta$ by any element of $k_{0}$ whose norm in $k$ is a $t$ th power. Since $N_{k_{0} / k}$ is onto (as a map from $k$ to itself), we have $N_{k_{0} / k}\left(\operatorname{Tr}_{d / k_{0}} \beta\right)=N_{k_{0} / k} \beta_{0}$, $\beta_{0} \in k_{0}$. That is, we can modify $\operatorname{Tr}_{d / k_{0}} \beta$ to be $\left(\beta_{0}\left(\operatorname{Tr}_{d / k_{0}} \beta\right)^{-1}\right) \operatorname{Tr}_{d / k_{0}} \beta=$ $\beta_{0} \in k$, since $\left.N\left(\beta_{0}\right) T r_{d / k_{0}} \beta\right)^{-1}$ is a $p$ th power.

LEMMA 10. We can find elements $u^{\prime}$ and $u$ such that:

(a) $u^{\prime}$ is a prime element of $K(x)$ and $u$ is a prime element of $D_{x_{s}}$

(b) $u^{\prime} \equiv u \bmod P^{s}$.

Proof. Let $u^{\prime}$ be any prime element of $K(x)$. Then $u^{\prime}$ commutes with $x_{s} \bmod P^{-n-m+s}$, and Lemma 4 plus an easy induction shows that there is a prime element $u$ of $D_{x_{s}}$ with $u \equiv x^{\prime} \bmod P^{s}$. $K(x))$.

Assume that $s$ is even, $s=2 s_{0}$. Define $H_{x}=G_{n_{0}}\left(G_{n_{0}-s_{0}} \cap D_{x_{s}}\right)(G \cap$

LEMMA 11. (a) If $n_{0}-s_{0}<j<n$, then $\left[H_{x}, H_{x}\right] \cap G_{j} /\left[H_{x}, H_{x}\right] \cap$ $G_{j+1}$ has as coset representatives the elements $1+\alpha u^{p}, \operatorname{Tr}_{k_{0} / k} \alpha=0$. When $j=n_{0}-s_{0}$, the quotient is trivial.

(b) If $z \in\left[H_{x}, H_{x}\right] \cap G_{n_{0}}$, then $\chi_{x}(z)=1$.

Proof. This is almost the same as the proof of Lemma 8; the modifications are easy. 
From now on, the analogy with the procedure in $\S 3$ is not nearly so close. It is true that we could now extend $\chi_{x}$ to a group $H_{x}$ (as in Theorem 5) and induce to $G$, and that the result would be an irreducible representation of $G$. The trouble is that we would not get enough representations of $G$ in this way. The way out of this difficulty is to vary the group $H_{x}$. The details, as we shall see, are notationally unpleasant.

We begin by analyzing the characters $\chi_{x}$ and $\chi_{x_{s}}$ more closely. Define the additive character $\psi^{(s)}$ on $K_{x_{s}}$ as was done in $\S 3$, and let $\omega$ be a 1-dimensional representation of $D_{x_{s}} \cap G$ which agrees with $\chi_{x_{s}}$ on $D_{x_{s}} \cap G_{n_{0}}$. We may extend $\omega$ to $G_{n_{0}}\left(D_{x_{s}} \cap G\right)$ by letting it equal $\chi_{x_{s}}$ on $G_{n_{0}}$. Then $\omega$ is trivial on all commutators of $D_{x_{s}} \cap G_{n_{0}-s_{0}}$, and $\chi_{x} \omega^{-1}$ is trivial on $G_{n-s}$. Hence if $\phi$ is any 1-dimensional representation of $D_{x_{s}} \cap G_{n_{0}-s_{0}}$ which agrees with $\chi_{x} \omega^{-1}$ on $D_{x_{s}} \cap G_{n_{0}}$, then we can extend $\chi_{x}$ to $G_{n_{0}}\left(D_{x_{s}} \cap G_{n_{0}-s_{0}}\right)$ by letting it equal $\phi \omega$ on $D_{x} \cap G_{n_{0}-s_{0}}$. There is an element $w \in D_{x_{s}}$ such that

$$
\phi(1+z)=\psi^{(s)} \circ \tau_{D_{x_{\varepsilon} / K\left(x_{s}\right.}(z w),}\left(z \in D_{x_{s}} \cap P^{n_{0}-s_{0}} .\right.
$$

We write $\phi=\chi_{w}^{(s)}$. In fact,

$$
w=\sum_{j=-n-m+s}^{\infty} \beta_{j} u^{j},
$$

and, if $x-x_{s}=\alpha^{\prime} \pi^{-n-m+s}+$ higher order terms, then a calculation like that in part (a) of Theorem 5 shows that

$$
\beta_{-n-m+s}=T r_{k / k_{0}}\left(\alpha^{\prime}\right) \text {. }
$$

From Lemma 9, $\beta_{-m-n+s} \in k$.

LEMMA 12. (a) There are $q^{s_{0}}$ orbits of the $\chi_{w}^{(s)}$ under the action of $D_{x_{\circ}} \cap G_{n_{0}-s}$.

(b) If $3 s_{0} \geqq n_{0}$, then $w=\sum_{j=-n-m+s}^{\infty} \beta_{j} u^{j}$, with $\beta_{j} \in k$ for $j \leqq$ $-n_{0}-m$

(c) If $3 s_{0}<n_{0}$, let $t$ be the smallest integer with $2 t \geqq n_{0}-s_{0}$. Then $\beta_{j} \in K \cap k_{1}$ for $j \leqq-n_{0} \neq s_{0}-m-t$.

Proof. (a) The element $\beta_{-n+s-m} u^{-m-n+s}$ generates a totally ramified field of degree $p$ over $K\left(x_{s}\right)$. It follows (as in Lemma 1) that the elements of $D_{x_{s}} \cap G_{n_{0}-s} / D_{x_{s}} \cap G_{n_{0}-s_{0}}$ commuting with $\chi_{w}^{\prime}$ are (congruent $\bmod P^{n_{0}-s_{0}}$ to) elements of $K\left(x_{s}, w\right)$, and there are $q^{s_{0}}$ such elements. The orbit calculation follows easily.

(b) Let $u, u^{\prime}$ be as in Lemma 10. If $y \in D_{x_{s}} \cap G_{n_{0}-s_{0}}$ and $v^{\prime} \in$ $K(x) \cap G_{s_{0}}$, then $\chi_{x}\left(v^{\prime} v v^{\prime-1} v^{-1}\right)=1$, from Lemma 11. But $\chi_{x_{s}}\left(v^{\prime} y v^{\prime-1} y^{-1}\right)=1$ as well. For if $y=1+y_{0}, v^{\prime}=1+v_{0}$, then 
But

$$
\chi_{x_{s}}\left(v^{\prime} y v^{\prime-1} y^{-1}\right)=\psi \circ \tau_{D / K} x_{8}\left(\sum_{j=0}^{\infty}(-1)^{j}\left(v_{0} y_{0} v_{0}^{j}-y_{0} v_{0}^{j+1}\right)\right)
$$

$$
\tau_{D / K}\left(x_{s} v_{0} y_{0}-x_{s} y_{0} v_{0}\right)=\tau_{D / K}\left(x_{s} v_{0} y_{0}-y_{0} x_{s} v_{0}\right)=0,
$$

while the other terms in the parentheses are in $P^{n-s}$, so that we can replace $x_{s}$ by $x$. (It is here that we need the estimate of the size of $s$.) As $v_{0}$ and $x$ commute, a similar calculation shows that the remaining terms have trace 0 .

Thus $\chi_{w}^{\prime}\left(v^{\prime} y v^{\prime-1} y^{-1}\right)=1$. Recall that $v^{\prime}$ is given by a polynomial in $u^{\prime}$; let $v$ be the corresponding polynomial in $u$. Then

$$
\chi_{w}^{\prime}\left(v y v^{-1} y^{-1}\right)=1,
$$

since $v y v^{-1} y^{-1}$ and $v^{\prime} y v^{\prime-1} y^{-1}$ agree modulo $G^{n-s}$. Since $\chi_{w}^{\prime}$ is trivial on these commutators, we must have $w \in K(u) \bmod P^{-m-n_{0}}$.

The proof of (c) is essentially the same as that of (b).

Note. Parts (b) and (c) of Lemma 12 may seem paradoxical. After all, we may vary $x-x_{s}$ freely on $P^{-m-n+s}$; how can we restrict the $\beta_{j}$ in any way? The answer is that the element $u \in D_{x_{s}}$ depends on $x$; varying $x$ means a new choice of $u$, and hence a different expression for $w$.

Define $\chi_{w}^{(s)}(y)=\psi^{(s)} \circ \tau_{D_{x_{s}} / K\left(x_{s}\right)}(y-1), y \in G_{n_{0}-s_{0}}$, if $w \in D_{x_{s}} \cap P^{-n-m+s}$, and let $H_{x, 0}=G_{n_{0}}\left(G_{n_{0}-s_{0}} \cap D_{x_{s}}\right)(G \cap K(x))$. We know that there are extensions of $\chi_{x}$ to $H_{x, 0}$. Let $\chi_{x, 0}$ be one, and let $\chi_{x, 0} \omega^{-1} \mid D_{x_{s}} \cap G_{n_{0}-s_{0}}=\chi_{w_{1}}^{(x)}$. Write $w_{1}=w_{1 ; 0}+w_{1 ; 1}$, where $w_{1 ; 0} \in K\left(x_{s}\right)$ and $w_{1 ; 1} \in P^{-m-n_{0}+1}$ or $P^{-m-n_{0}-s_{0}-t+1}$, depending on whether (b) or (c) of Lemma 12 applies. Choose one element from each of the orbits in Lemma 12(a); a typical element is $w=w_{1}+w_{b}, w_{b} \in P^{-m-n+s} \cap D_{x_{s}}$. For the $w_{1}$-orbit, we may, and do, take $w_{b}=0$.

Suppose that $v^{\prime} \in K(x) \cap G$, and $y \in D_{x_{s}} \cap G_{n_{0}-s_{0}}$. In investigating whether the induced representations we shall construct are irreducible, we need to know when $y v^{\prime}$ commutes with $\chi_{x ; 0} \cdot \chi_{w_{b}}^{(s)}$ on $D_{x_{s}} \cap G_{n_{0}-s_{0}}$. Write $z(\chi)$ for the conjugate action of $z$ on $\chi$. Since $v^{\prime}$ commutes with $\chi_{x ; 0}$, we have

$$
\begin{aligned}
\left(y v^{\prime}\right)\left(\chi_{x ; 0} \cdot \chi_{w_{b}}^{(s)}\right)\left(\chi_{x ; 0} \cdot \chi_{w_{b}}^{(s)}\right)^{-1} & =y\left(\chi_{x ; 0}\right) \cdot(y v)\left(\chi_{w_{b}}^{(s)}\right) \cdot\left(\chi_{x ; 0} \cdot \chi_{w_{b}}^{(s)}\right)^{-1} \\
& =y\left(\chi_{x ; 0} \cdot \omega^{-1}\right)\left(y v^{\prime}\right)\left(\chi_{w_{b}}^{(s)}\right)\left(\chi_{x ; 0} \cdot \omega^{-1} \cdot \chi_{w_{b}}^{(s)}\right)^{-1} \\
& =y\left(\chi_{w_{1}}^{(s)}\right) \chi_{\left(y v^{\prime} w_{b} v^{\prime-1} y^{-1}\right)} \cdot\left(\chi_{w_{1}}^{(s)} \cdot \chi_{w_{b}}^{(s)}\right)^{-1} \\
& =\chi_{\left(y w_{1 ;} y^{-1}\right)}^{(s)} \chi_{\left(y w_{1 ; 1} y^{-1}\right)}^{(s)} \chi_{y v^{\prime} w_{b} v^{\prime-1} y^{-1}}^{(s)} \chi_{-w_{1}-w_{b}}^{(s)} .
\end{aligned}
$$

It is easy to check that $y$ commutes with $\chi_{w_{1 ; 1}}^{(s)}$; for instance, if Lemma 12(b) applies, then

$$
y w_{1: 1} y^{-1}-w_{1 ; 1} \in P^{-m-n_{0}+1+n_{0}-s_{0}}=P^{-m-s_{0}+1}
$$


and thus $\chi_{y w_{1 ; 1} y^{-1}-w_{1 ; 1}}^{(s)}$ is trivial on $D_{x_{s}} \cap G_{n_{0}-s_{0}}$. Similarly, $\chi_{v^{\prime} w_{1 ; 0} v^{\prime-1}}^{(s)}=$ $\chi_{w_{1 ; 0}}^{(s)}$. We can therefore write the last expression as

$$
\chi_{y v^{\prime} w_{1} ; v^{\prime}-1}^{(s)} \chi^{-1} \chi_{\left(y v^{\prime} w_{b} v^{\prime-1} y^{-1}\right)}^{(s)} \chi_{-w_{1} ; 0}^{(s)} \chi_{-w_{b}}^{(s)} .
$$

Thus $y v^{\prime}$ commutes with $\chi_{x ; 0} \cdot \chi_{w_{b}}^{(s)}$ iff $y v^{\prime}$ commutes with $w_{1 ; 0}+w_{b}$ (modulo a sufficiently high power of $\rho$ ).

LEMMA 13. Let $u_{1}=\left(w_{1 ; 0}+w_{b}\right)^{r_{1}} \pi^{-m r_{2}}$ be a prime element in $K\left(w_{1 ; 0}+w_{b}, x_{s}\right)$, so that $u_{1}=\sum_{j=1}^{\infty} \varepsilon_{j} u^{j}$, with $\varepsilon_{j} \in k$ for $1 \leqq j \leqq n_{0}-s$ and $\varepsilon_{j} \in k_{0}$ generally. Let $u_{1}^{\prime}=\sum_{j=1}^{\infty} \varepsilon_{j}\left(u^{\prime}\right)^{j}$. Then elements in $G \cap K\left(u_{1}^{\prime}\right)$ commute with the representation on $G_{n_{0}}\left(G_{n_{0}-s_{0}} \cap D_{x_{s}}\right)$ given by $\chi_{s}$ on $G_{n_{0}}$ and extended (as indicated above) by $\chi_{w}^{(s)}, w=w_{1 ; 0}+w_{1 ; 1}+w_{b}$.

Let $H_{x ; b}=G_{n_{0}}\left(G_{n_{0}-s_{0}} \cap D_{x_{s}}\right)\left(G \cap K\left(u_{1}^{\prime}\right)\right)$. The 1-dimensional representations described above have exactly $q^{n_{0}-s_{0}-1}$ extensions to $H_{x ; b}$.

Proof. We checked above that the elements in $G \cap K\left(u_{1}^{\prime}\right)$ commute with the given representation; they also commute with other. Therefore the representation extends. The last statement holds because $\left[H_{x ; b}: G_{n_{0}}\left(G_{n_{0}-s_{0}} \cap D_{x_{s}}\right)\right]=q^{n_{0}-s_{0}-1}$.

We are now (finally!) ready to describe the irreducible representations of $G$ containing $\chi_{x}$.

THEOREM 6. Let $\chi_{x ; b ; a}$ be one of the 1-dimensional representations of $H_{x ; b}$ described in Lemma 13. (Recall that one chooses exactly one $w=w_{1}+w_{b}$ from each of the orbits in Lemma 12(a).) Let $\rho_{x ; b ; a}=$ $\operatorname{Ind}_{H_{x ; b} \rightarrow G} \chi_{x ; b ; a}$. Then:

(a) $\rho_{x ; b ; a}$ is irreducible;

(b) the $\rho_{x: b ; a}$ are mutually disjoint;

(c) the $\rho_{x ; b ; a}$ exhaust the irreducible representations of $G$ containing $\chi_{x}$.

Proof. For (a), let $H_{x ; w}^{\prime}=G_{n_{0}}\left(G_{n_{0}-s} \cap D_{x_{s}}\right)\left(G \cap K\left(u_{1}^{\prime}\right), J_{x}=G_{n_{0-s}} D_{x_{s}}\right.$. The elements of $G$ which fix $\chi_{x} \mid G_{n_{0}}$ are all in $H_{x ; w}^{\prime}$, and $H_{x ; w}^{\prime}$ normalizes $H_{x ; w}$. Moreover, the coset representatives for $H_{x ; w}^{\prime} / H_{x ; w}$ can all be chosen from among coset representatives of $J_{x} / J_{x} \cap G_{n_{0}-s_{0}}$. We need only show, therefore, that any element of $J_{x}$ (modulo $J_{x} \cap G_{n_{0}-\varepsilon_{0}}$ ) which commutes with $\chi_{x ; w ; a}$ lies in $H_{x ; w}$. From Lemma 11, there are exactly $q^{n_{0}-s_{0}}$ such elements, and elements of $H_{x ; w}$ account for them. Thus $\rho_{x ; w ; a}$ is irreducible.

The proof of (b) is nearly identical; we need to show that no element of $H_{x ; w}^{\prime}$ can transform one $\chi_{x ; b ; a}$ into a $\chi_{x ; b^{\prime} ; a^{\prime}}$, and this is evident because $w, w^{\prime}$ are in different orbits. Finally, (c) is the usual counting argument.

Theorems 1-6 solve the problem of finding the irreducible repre- 
sentations of $G$, except for some minor details to be dealt with in the next section.

5. We still need to remove the assumptions on the parity of $n$ and $s$. As the procedure is the same as in [1] and [4], we omit proofs. (Some of the counting arguments above must be modified; this, too, is not hard.)

If $n$ is even, but $n-s$ is odd, let $n_{0}=1+(n / 2)$, and let $\left(H_{0}\right)_{x}$ or $\left(H_{0}\right)_{x ; w}$ be the group described in $\S 2$ or 3 (so that if, e.g., $K\left(x_{1}\right)=K(x)=p^{2}$, then $\left(H_{0}\right)_{x}=G_{n_{0}}(K(x) \cap G)$. We shall write $\left(H_{0}\right)_{x}$ in what follows, but the remarks apply equally to $\left(H_{0}\right)_{x ; w}$. Consider the bilinear form

$$
B_{x}:(\alpha, \beta) \longmapsto \chi_{x}\left(1+\alpha \pi^{n_{0}-1}\right)\left(1+\beta \pi^{n_{0}-1}\right)\left(1+\alpha \pi^{n_{0}-1}\right)^{-1}\left(1+\beta \pi^{n_{0}-1}\right)^{-1}
$$

taking $d \times d$ to the unit circle. It is antisymmetric, and the radical is $\left\{\beta: K\left(x_{1}\right)\right.$ contains an element $\sum_{j=n_{0}-1}^{\infty} \beta_{j} \pi^{j}$ with $\left.\beta_{n_{0}-1}=\beta\right\}$. Therefore the elements $1+\beta \pi^{n_{0}-1}$ with $\beta \in \operatorname{Rad}\left(B_{x}\right)$ are already contained in $\left(H_{0}\right)_{x}$. Let $h$ be a maximal isotropic subspace of $B_{x}$; we let $H_{x}$ be the group generated by $\left(H_{0}\right)_{x}$ and the elements $\left(1+\beta \pi^{n_{0}-1}\right)$ with $\beta \in h$. Two extensions of $\chi_{x}$ to $H_{x}$ are equivalent if they are equivalent on $\left(H_{0}\right)_{x}$. The representations $\rho_{x ; a}=\operatorname{Ind}_{H_{x \rightarrow G} \chi_{x ; a}}$ are irreducible and exhaust the representations of $G$ whose restrictions to $G_{n_{0}}$ contain $\chi_{x}$, and inequivalent $\chi_{x ; a}$ give rise to inequivalent $\rho_{x ; a}$.

If $n-s$ is even, the procedure is similar; let $n_{0}-s_{0}=(n-s) / 2+1$ and consider a maximal isotropic subspace $h^{\prime}$ of

$$
\begin{aligned}
B_{x}^{\prime}:(\alpha, \beta) \longmapsto & \chi_{x-x_{s}}\left(\left(1+\alpha u^{n_{0}-s_{0}-1}\right)\left(1+\beta u^{n_{0}-s_{0}-1}\right)\right. \\
& \left.\times\left(1+\alpha u^{n_{0}-s_{0}-1}\right)^{-1}\left(1+\beta u^{n_{0}-s_{0}-1}\right)^{-1}\right) ;
\end{aligned}
$$

add the elements $\left(1+\alpha u^{n_{0}-s_{0}-1}\right), \alpha \in h^{\prime}$, to $\left(H_{0}\right)_{x}$, and call extensions of $\chi$ to $\left(H_{0}\right)_{x}$ equivalent if they agree on $H_{0}$.

There is another description of this procedure, in which if, e.g., $n$ is odd, one tensors $\chi_{x ; a}$ on $\left(H_{0}\right)_{x}$ with a Heisenberg-like representation of $G_{n_{0}-1}$; see [4].

We also need to extend representations from $G$ to $D^{x}$. The method is generally like that in [1]; here, for example, is a description for the case $\left[K\left(x_{1}\right): K\right]=p^{2}$. We may assume that the maximal unramified extension in $K(x)$ is contained in $K_{1}$. Let $x^{\prime}$ be a prime element of $K(x)$, and let

$$
J_{x}=\left\{\left(x^{\prime}\right)^{j}: j \in Z\right\} \cdot\left\{k_{1} \cap K^{x}(x)\right\} .
$$

Then conjugation by elements of $J_{x}$ fixes $\chi_{x ; a}$ on $H_{x}$. Tensor the $\chi_{x ; a}$ with representations of $J_{x}$ and induce to find the irreducible representations of $D^{x}$. 
The case considered in $\S 4$ is somewhat different; we merely sketch the argument, since the result is not quite satisfactory. We use the notation of $\S 4$. Let $\rho_{x ; b ; a}$ be induced from $\chi_{x ; b ; a}$ on $H_{x ; b}$, and let $F$ be the trace character of $\chi_{x ; ; ; a}$. We note that $u_{1}^{\prime}$ normalizes $H_{x ; b}$. Let $y \in G$. If no $G$-conjugate of $y$ lies in $H_{x ; b}$, then no $G$-conjugate of $u_{1}^{\prime} y\left(u_{1}^{\prime}\right)^{-1}$ lies in $H_{x ; b}$; hence $F(y)=F\left(u_{1}^{\prime} y u_{1}^{\prime-1}\right)=0$. On the other hand, it is not hard to see that if $y \in H_{x ; b}$, then there is a prime element $v=\sum_{j=1}^{\infty} \eta_{j} \pi^{j}, \eta_{1}=1$, such that $F\left(v y v^{-1}\right)=F(y)$. (For instance, if $y \in G_{n_{0}}$, then $\chi_{\left(u^{\prime}\right)-1} u_{u^{\prime}}=\chi_{x}$, and so $F\left(u^{\prime} y\left(u^{\prime}\right)^{-1}\right)=F(y)$.) Since $v u^{\prime} \in G$, we conclude that, e.g., conjugation by $u_{1}^{\prime}$ fixes $F$. Thus one can extend $\rho_{x: b ; a}$ to $J_{x}$. Now one proceeds as in [1]. It would, of course, be preferable to have a concrete description to $J_{x}$.

The procedure for constructing representations described in this paper also applies to division algebras $D$ whose index $m$ is the product of two primes. (If neither prime is $p$, then, of course, we are in the situation of [1] or [3].) There is some difference in the consideration of cases because the behavior of $T r_{k_{0} / k}$ depends on whether or not $\left[k_{0}: k\right]$ is prime to $p$. The procedure also applies to some representations in the case where $m$ is a power of $p$; for instance, if $m=p^{r}$ and $\left[K\left(x_{1}\right): K(x)\right]=p^{r-1}$, one can find the representations of $G$ containing $\chi_{x}$ by methods like those of $\S \S 3$ and 4 .

The general case is murkier. The problems with extending the procedures of $\S \S 3$ and 4 are twofold: the approximation lemma analogous to Lemmas 7 and 10 becomes more difficult to prove, and one needs to deal with certain finite-dimensional representations of $H_{x}$ which are hard to describe. I hope to return to this topic in future papers.

Two other matters deserve mention. In [6], Langlands predicted a correspondence between representations of $D$ and certain representations of the Weil group of $K$. This correspondence has been verified in the tamely ramified case (see [5]). It is of considerable interest to know whether the correspondence holds more generally.

Finally, there is the question of finding the characters of the representations. In the tamely ramified case, the computations are found in [4]. The wildly ramified case seems much harder.

\section{REFERENCES}

1. L. Corwin, Representations of division algebras over local fields, Advances in Math., 13 (1974), 259-267.

2. L. Corwin and $\mathrm{R}$. Howe, Computing characters of tamely ramified p-adic division Algebras, Pacific J. Math., 73 (1977), 461-477.

3. R. Howe, Representation theory for division algebras over local fields (tamely ramified case), Bull. Amer. Math. Soc., 17 (1971), 1063-1066.

4. - Kirillov theory for compact p-adic groups, Pacific J. Math., 73 (1977), 365-381. 
5. H. Koch, preprint.

6. R. P. Langlands, Problems in the Theory of Automorphic Forms, in Lectures on Modern Analysis \& Applications, Springer Lecture Notes, Vol. 170 (1970).

7. G. W. Mackey, On induced representations of groups, Amer. J. Math., 73 (1951), 576-592.

8. A. Weil, Basic Number Theory, Springer Verlag, Heidelberg-New York, 1973.

Received September 25, 1979 and in revised form April 15, 1981. This research was supported by NSF Grant NC578-02715, and was performed in part while the author was on sabbatical leave at the University of California, Berkeley.

Rutgers University

New Brunswick, NJ 08903 


\section{PACIFIC JOURNAL OF MATHEMATICS}

\section{EDITORS}

DONALD BABBITT (Managing Editor)

J. DUGUNDJI

University of California

Los Angeles, California 90024

Hugo Rossi

University of Utah

Salt Lake City, UT 84112

C. C. Moore and Arthur Agus

Department of Mathematics

University of Southern California

Los Angeles, California 90007

R. FinN and J. Milgram

Stanford University

Stanford, California 94305

University of California

Berkeley, CA 94720

\section{ASSOCIATE EDITORS}
R. ARNES
E. F. BeCKenBaCH
B. H. NEUMANN
F. WoLF
K. YoshidA

\section{SUPPORTING INSTITUTIONS}

UNIVERSITY OF ARIZONA

UNIVERSITY OF BRITISH COLUMBIA

CALIFORNIA INSTITUTE OF TECHNOLOGY

UNIVERSITY OF CALIFORNIA

MONTANA STATE UNIVERSITY

UNIVERSITY OF NEVADA, RENO

NEW MEXICO STATE UNIVERSITY

OREGON STATE UNIVERSITY
UNIVERSITY OF OREGON

UNIVERSITY OF SOUTHERN CALIFORNIA

STANFORD UNIVERSITY

UNIVERSITY OF HAWAII

UNIVERSITY OF TOKYO

UNIVERSITY OF UTAH

WASHINGTON STATE UNIVERSITY

UNIVERSITY OF WASHINGTON 


\section{Pacific Journal of Mathematics}

\section{Vol. 101, No. $1 \quad$ November, 1982}

Natália Bebiano, On the evaluation of permanents $\ldots \ldots \ldots \ldots \ldots \ldots \ldots \ldots$

David Borwein and Bruce Brigham Watson, Tauberian theorems between

the logarithmic and Abel-type summability methods $\ldots \ldots \ldots \ldots \ldots \ldots 11$

Leo George Chouinard, II, Hermite semigroup rings $\ldots \ldots \ldots \ldots \ldots \ldots \ldots$

Kun-Jen Chung, Remarks on nonlinear contractions $\ldots \ldots \ldots \ldots \ldots \ldots . \ldots 4$

Lawrence Jay Corwin, Representations of division algebras over local

fields. II ......................................... 49

Mahlon M. Day, Left thick to left lumpy—a guided tour $\ldots \ldots \ldots \ldots \ldots 71$

M. Edelstein and Mo Tak Kiang, On ultimately nonexpansive

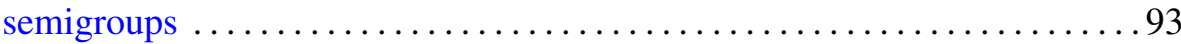

Mary Rodriguez Embry, Semigroups of quasinormal operators . ........ 103

William Goldman and Morris William Hirsch, Polynomial forms on

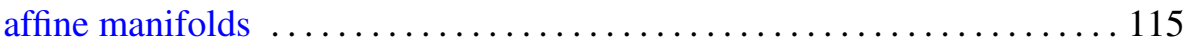

S. Janakiraman and T. Soundararajan, Totally bounded group topologies

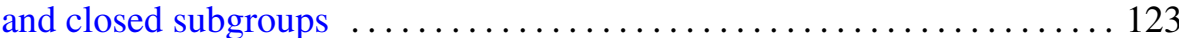

John Rowlay Martin, Lex Gerard Oversteegen and Edward D.

Tymchatyn, Fixed point set of products and cones $\ldots \ldots \ldots \ldots \ldots \ldots 133$

Jan van Mill, A homogeneous Eberlein compact space which is not metrizable ........................................ 141

Steven Paul Plotnick, Embedding homology 3-spheres in $S^{5} \ldots \ldots \ldots \ldots 147$

Norbert Riedel, Classification of the $C^{*}$-algebras associated with minimal rotations

Benedict Seifert, Combinatorial and geometric properties of weight systems of irreducible finite-dimensional representations of simple split Lie algebras over fields of 0 characteristic

James E. Simpson, Dilations on locally convex spaces

Paolo M. Soardi, Schauder bases and fixed points of nonexpansive mappings

Yoshio Tanaka, Point-countable $k$-systems and products of $k$-spaces

Fausto A. Toranzos, The points of local nonconvexity of starshaped sets . . . 209

Lorenzo Traldi, The determinantal ideals of link modules. I . . . . . . . . 215

P. C. Trombi, Invariant harmonic analysis on split rank one groups with applications

Shinji Yamashita, Nonnormal Blaschke quotients 